\title{
A Faster and More Space-Efficient Algorithm for Inferring Arc-Annotations of RNA Sequences Through Alignment
}

\author{
Jesper Jansson ${ }^{1}$, See-Kiong $\mathrm{Ng}^{2}$, Wing-Kin Sung ${ }^{1}$, and Hugo Willy ${ }^{1}$ \\ 1 Department of Computer Science, National University of Singapore \\ 3 Science Drive 2, Singapore 117543 \\ \{jansson, ksung, hugowill\}@comp.nus.edu.sg \\ 2 Institute for Infocomm Research, 21 Heng Mui Keng Terrace, Singapore \\ skng@i2r.a-star.edu.sg
}

\begin{abstract}
This paper considers the problem of inferring the optimal nested arc-annotation of a sequence given another nested arc-annotated sequence by maximizing the weighted alignment between the bases and arcs in the two sequences. The problem has a direct application in predicting the secondary structure of an RNA sequence given a closely related sequence whose secondary structure is already known. The currently most efficient algorithm for this problem requires $O\left(\mathrm{~nm}^{3}\right)$ time and $O\left(n m^{2}\right)$ space where $n$ is the length of the sequence with known arc-annotation while $m$ is the length of the sequence to be inferred. We present an improved algorithm which runs in $\min \left\{O\left(n m^{2} \log n\right)\right.$, $\left.O\left(n m^{3}\right)\right\}$ time and $\min \left\{O\left(m^{2}+m n\right), O\left(m^{2} \log n\right)\right\}$ space. The time improvement is achieved by applying sparsification to the dynamic programming algorithm, while the space is reduced to a more practical quadratic complexity by using a Hirschberg-like traceback technique together with a simple compression.
\end{abstract}

\section{Introduction}

Recent research shows that RNA functions as catalysts and regulators in nucleic acid processing and gene expression in addition to its commonly known intermediary role in DNA transcription and translation process. It is generally known that much of RNA's functionalities depend on its structural features. Unfortunately, although massive amount of sequence data are continuously generated, the number of known RNA structures is still very limited since experimental methods, such as NMR and Crystallography, require expertise and long experimental time. Therefore, computational methods for predicting RNA structure are very useful.

There exist a number of computational approaches to predict the structure of RNA in the literature. Basically, they can be classified into three categories: Energy Minimization, Comparative, and Structure Inferring methods. The first approach tries to compute the structure of an RNA molecule which has the lowest free energy. Representatives of this approach are the methods of Nussinov et al [16] and Zuker et al [15,20,21]. Since the current energy model is not 
accurate enough and RNA may not fold into the lowest energy structure, the prediction accuracy of this method is usually not high. For the Comparative method, we are given a number of RNA sequences which are expected to have similar structure called the homologous sequences. By aligning those RNA sequences, we compute the consensus structure. Representatives of this approach include Maximum Weighted Matching (MWM) [3, 18] and Stochastic Context Free Grammars (SCFGs) [8,7,17]. The Comparative approach is currently the best way to predict RNA structures $[9,12]$. However, when the number of homologous sequences is not large enough, the accuracy can be low. If we only have a few homologous RNA sequences where the structure of one of the sequences is known, the RNA structure can be predicted using the Structure Inferring method $[2,19]$. Consider two sequences $S_{1}$ and $S_{2}$ of length $n$ and $m$. Assuming that the secondary structure of $S_{1}$ is known, this method infers the secondary structure of $S_{2}$ by aligning $S_{1}$ and $S_{2}$. Bafna et al [2] propose a dynamic programming solution to this problem and solve it using $O\left(n^{2} m^{2}+n m^{3}\right)$ time and $O\left(n^{2} m^{2}\right)$ space. Zhang [19] improves their result and gives an algorithm which runs in $O\left(\mathrm{~nm}^{3}\right)$ time and $O\left(\mathrm{~nm}^{2}\right)$ space. In this paper, we further improve the running time of the inference algorithm to $\min \left\{O\left(n m^{2} \log n\right), O\left(n m^{3}\right)\right\}$ and at the same time bring down the space requirement to $\min \left\{O\left(m^{2}+m n\right), O\left(m^{2} \log n\right)\right\}$.

Our improvement in the running time stems from sparsification. We observe that the entries in every row and every column in the dynamic programming tables are monotonically increasing, enabling us to calculate less entries in the tables without losing any information. We also designed a new recursive dynamic programming algorithm that gives a better worst-case space requirement in the case of computing only the score of the alignment of $S_{1}$ and $S_{2}$. Finally, by incorporating the latter into an algorithm similar to Hirschberg's traceback [10] together with a simple compression method, we can recover the optimal inferred structure from the table within the stated reduced space complexity. Note that the space improvement is critical in our application since currently the length of a typical RNA sequence used in lab experiments is around $3 \mathrm{~K}$ to $5 \mathrm{~K}$ bases. Assuming that $n \approx m$, the memory requirement of an $O\left(n m^{2}\right)$ space algorithm could easily reach over tens of gigabytes. This memory requirement is not impossible to meet but it is highly impractical.

This paper is organized as follows. Section 2 contains the formal statement of the problem with some basic definitions. Section 3 presents the algorithm and is divided into three parts. The first part presents the original algorithm given in [19], noting the bottleneck of the computation. The following two parts present our techniques to improve the running time of the algorithm. The Hirschberglike traceback algorithm is described in Section 4. Finally, Section 5 concludes this paper with some possible extensions of the problem.

\section{Preliminaries}

We use a slightly different notation from the one in [19] where the secondary structure of the first sequence is represented as a tree. Each internal node in the 
tree represents a base pair and the bases in the loop created by the base pair are the children of the node.

In our algorithm, we represent an RNA sequence and its secondary structure information using the arc-annotated sequence [4]. Consider a sequence $S$ over a fixed alphabet $\Sigma=\{A, C, G, U\}$. We define $S[i]$ to be the $i^{\text {th }}$ character in $S$ and $S[i . . j]$ to be the substring of $S$ in positions between $i$ and $j$ (inclusive). For any $x \in \Sigma$, let Complement $(x)$ be the complementary base of $x$ based on the Watson-Crick base pairing. For example Complement $(A)$ is $U$ and Complement $(G)$ is $C$. An unordered pair of positions $(i, j)$, where $i<j$, indicates that $S[i]$ and $S[j]$ form a base pair in the RNA structure. Such pair is called an arc. For RNA sequences, it is required that $S[j]=\operatorname{Complement}(S[i])$ and vice versa. A set $P$ of arcs is called an arc-annotation, and the pair $(S, P)$ is called an arc-annotated sequence. Arc-annotated sequences are well-studied $[1,4,6,11,13,14,19]$ and are commonly used in computational biology to represent the structure of RNA and protein sequences. Since we are considering RNA secondary structures, we assume that the RNA sequences we are dealing with do not have any pseudoknots. The corresponding arc-annotation construct for such RNA structures is the nested arc-annotation $[1,11,13,14]$ where, given two arcs, either one is within the other, or they are completely disjoint $\left(\forall\left(i_{1}, j_{1}\right),\left(i_{2}, j_{2}\right) \in P, i_{1} \in\left[i_{2}, j_{2}\right] \Leftrightarrow j_{1} \in\left[i_{2}, j_{2}\right]\right)$. For any arc $u \in P$, we denote $u_{l}$ and $u_{r}$ to be the left and the right endpoints of $u$, respectively. The size of an $\operatorname{arc} u$ is denoted by $|u|=u_{r}-u_{l}+1$. We say that position $i$ is free if $i$ is not an endpoint of any $\operatorname{arc}$ in $P$. A position $i$ is covered by an $\operatorname{arc} u$ if $u_{l}<i<u_{r}$ and there exist no other arc $u^{\prime}$ such that $u_{l}^{\prime}<i<u_{r}^{\prime}$. The set of all positions covered by $u$ is called the arc cover of $u$, denoted by $C(u)$.

Given two arc-annotated sequences $\left(S_{1}, P_{1}\right)$ and $\left(S_{2}, P_{2}\right)$, we can define the similarity of the sequences by aligning the bases and the arcs in them. We need to define a scoring function for each type of alignment. Let $\chi$ be the function to score the alignment of unpaired bases in the two sequences where, for $a, b \in\{A, C, G, U, \sqcup\}, \chi(a, b)=\beta$ if $a=b$ and 0 otherwise (' $\sqcup$ ' denotes a blank character). For any arc $u$, which represents paired bases in the RNA structure, let $\delta$ be a scoring function for arcs alignment whose value is defined as:

$$
\delta\left(\left(S_{1}\left[u_{l}\right], S_{1}\left[u_{r}\right]\right),\left(S_{2}[j], S_{2}\left[j^{\prime}\right]\right)\right)=\left\{\begin{array}{cc}
\alpha_{1} & \text { if } S_{1}\left[u_{l}\right]=S_{2}[j] \text { and } S_{1}\left[u_{r}\right]=S_{2}\left[j^{\prime}\right] \\
\alpha_{2} & \text { if } S_{1}\left[u_{l}\right] \neq S_{2}[j] \text { and } S_{1}\left[u_{r}\right] \neq S_{2}\left[j^{\prime}\right] \\
& \text { but } S_{2}[j]=C o m p l e m e n t\left(S_{2}\left[j^{\prime}\right]\right) \\
-\infty & \text { otherwise }
\end{array}\right.
$$

$\beta, \alpha_{1}$, and $\alpha_{2}$ are positive integer constants. Usually the parameters are set such that $\beta \leq \alpha_{2} \leq \alpha_{1}$ which reflects that an arc-alignment $\left(\alpha_{1}\right.$ or $\left.\alpha_{2}\right)$ takes precedence over single base alignment $(\beta)$. Moreover, an arc alignment with exactly the same base pairs should score higher $\left(\alpha_{1}\right)$ since both bases and their arc are aligned. One can also have constraints on the arc width. For example, when $\left|j-j^{\prime}\right|$ is less than some minimum arc width parameter, we can define $\delta=-\infty$. Now given the definition of the arc annotation and the scoring functions, we formally state our problem (slightly altered from the one in [19]) as follows. 
The Weighted Largest Common Substructure(WLCS) of two arc annotated sequences $\left(S_{1}, P_{1}\right)$ and $\left(S_{2}, P_{2}\right)$ is defined as the maximum weighted alignment between $S_{1}$ and $S_{2}$ where free bases are aligned to free bases and arcs are aligned to arcs. The WLCS score is then defined as the sum of all bases and arcs alignment scores. The problem we address in this paper is: Given a nested arc-annotated sequence $\left(S_{1}, P_{1}\right)$ and a plain sequence $S_{2}$, infer the nested arcannotation $P_{2}$ for $S_{2}$ that maximizes their WLCS score.

\section{Algorithm Description}

This section reviews Zhang's algorithm (presented in [19]) for inferring the RNA secondary structure $P_{2}$ for $S_{2}$ that maximizes the WLCS score between $\left(S_{1}, P_{1}\right)$ and $\left(S_{2}, P_{2}\right)$. Let $\left|S_{1}\right|=n$ and $\left|S_{2}\right|=m$. Let $D P_{\left(i, i^{\prime}\right)}\left[j, j^{\prime}\right]$, where $1 \leq i \leq$ $i^{\prime} \leq n$ and $1 \leq j \leq j^{\prime} \leq m$, denotes the score of the weighted largest common substructure between $\left(S_{1}\left[i . . i^{\prime}\right], P_{1}\right)$ and $S_{2}\left[j . . j^{\prime}\right]$. Note that $D P_{\left(i, i^{\prime}\right)}\left[j, j^{\prime}\right]=0$ whenever $i>i^{\prime}$ or $j>j^{\prime}$. Zhang presented an algorithm which runs in $\mathrm{O}\left(n m^{3}\right)$ time and uses $\mathrm{O}\left(\mathrm{nm}^{2}\right)$ space based on a two-step dynamic programming. Given an arc $u$, the first step computes the value of $D P$ for the arc-cover of $u$ i.e. computes $D P_{\left(u_{l}+1, u_{r}-1\right)}\left[j, j^{\prime}\right]$ for all $1 \leq j \leq j^{\prime} \leq m$. Then, the next step computes the value of $D P$ for the whole arc $u$, that is $D P_{\left(u_{l}, u_{r}\right)}\left[j, j^{\prime}\right]$ for all $1 \leq j \leq j^{\prime} \leq m$. Below are the three equations in [19] to compute the two steps in the algorithm. Please refer to the paper for the proofs.

Lemma 1. (Lemma 4 in [19]) If either $i^{\prime}$ is free or $i^{\prime}$ is an endpoint of an arc whose other endpoint is not in $\left[i . . i^{\prime}-1\right]$,

$$
D P_{\left(i, i^{\prime}\right)}\left[j, j^{\prime}\right]=\max \left\{\begin{array}{l}
D P_{\left(i, i^{\prime}-1\right)}\left[j, j^{\prime}-1\right]+\chi\left(S_{1}\left[i^{\prime}\right], S_{2}\left[j^{\prime}\right]\right), \\
D P_{\left(i, i^{\prime}-1\right)}\left[j, j^{\prime}\right]+\chi\left(S_{1}\left[i^{\prime}\right], \sqcup\right), \\
D P_{\left(i, i^{\prime}\right)}\left[j, j^{\prime}-1\right]+\chi\left(\sqcup, S_{2}\left[j^{\prime}\right]\right)
\end{array}\right.
$$

Lemma 2. (Lemma 5 in [19]) For any arc $u \in P_{1}$ and $i<u_{l}$,

$$
D P_{\left(i, u_{r}\right)}\left[j, j^{\prime}\right]=\max _{j-1 \leq j^{\prime \prime} \leq j^{\prime}}\left\{D P_{\left(i, u_{l}-1\right)}\left[j, j^{\prime \prime}\right]+D P_{\left(u_{l}, u_{r}\right)}\left[j^{\prime \prime}+1, j^{\prime}\right]\right\}
$$

Lemma 3. (Lemma 3 in [19]) For any arc $u \in P_{1}$,

$$
D P_{\left(u_{l}, u_{r}\right)}\left[j, j^{\prime}\right]=\max \left\{\begin{array}{l}
D P_{\left(u_{l}+1, u_{r}-1\right)}\left[j+1, j^{\prime}-1\right]+ \\
\delta\left(\left(S_{1}\left[u_{l}\right], S_{1}\left[u_{r}\right]\right),\left(S_{2}[j], S_{2}\left[j^{\prime}\right]\right)\right), \\
D P_{\left(u_{l}+1, u_{r}-1\right)}\left[j, j^{\prime}\right] \\
D P_{\left(u_{l}, u_{r}\right)}\left[j+1, j^{\prime}\right], \\
D P_{\left(u_{l}, u_{r}\right)}\left[j, j^{\prime}-1\right]
\end{array}\right.
$$

Definition 1. If $i^{\prime}$ is free or $i^{\prime}$ is a right endpoint of an arc whose left endpoint is not in $\left[i . i^{\prime}\right]$, then given the table $D P_{\left(i, i^{\prime}-1\right)}, D P_{\left(i, i^{\prime}\right)}$ can be computed by using Lemma 1. We define the computation of $D P_{\left(i, i^{\prime}\right)}$ from $D P_{\left(i, i^{\prime}-1\right)}$ as the operation $\operatorname{EXTEND}\left(D P_{\left(i, i^{\prime}-1\right)}\right)$. 
$W L C S\left(S_{1}, P_{1}, P_{2}\right)$

For every arc $u \in P_{1}$ from the innermost to the outermost, left to right,

Step 1 : Compute $D P_{\left(u_{l}+1, u_{r}-1\right)}$ as follows.

For every $i \in C(u)$ in increasing order,

- if $i$ is free, compute $D P_{\left(u_{l}+1, i\right)}$ by $\operatorname{ExTEND}\left(D P_{\left(u_{l}+1, i-1\right)}\right)$.

- if $i=v_{r}$ for some arc $v$, compute $D P_{\left(u_{l}+1, i\right)}$ by $\operatorname{MERGE}\left(D P_{\left(u_{l}+1, v_{l}-1\right)}, D P_{\left(v_{l}, v_{r}\right)}\right)$.

- if $i=v_{l}$, do nothing.

Step 2 : Compute $D P_{\left(u_{l}, u_{r}\right)}$ by $\operatorname{ARC-MATCH}\left(D P_{\left(u_{l}+1, u_{r}-1\right)}\right)$.

Fig. 1. The algorithm from [19] described in terms of EXTEND, MERGE and ARCMATCH operations.

Definition 2. Consider any arc s. The operation $\operatorname{MERGE}\left(D P_{\left(i, s_{l}-1\right)}, D\right.$ $\left.P_{\left(s_{l}, s_{r}\right)}\right)$ is defined to be the computation of the table $D P_{\left(i, s_{r}\right)}$ given $D P_{\left(i, s_{l}-1\right)}$ and $D P_{\left(s_{l}, s_{r}\right)}$ using Lemma 2.

Definition 3. Consider any arc s. The operation ARC-MATCH $\left(D P_{\left(s_{l}+1, s_{r}-1\right)}\right)$ is defined to be the computation of the table $D P_{\left(s_{l}, s_{r}\right)}$ given $D P_{\left(s_{l}+1, s_{r}-1\right)}$ using Lemma 3.

Fig. 1 describes the procedure $W L C S\left(S_{1}, P_{1}, S_{2}\right)$ which computes $D P_{(1, n)}\left[j, j^{\prime}\right]$ for all $1 \leq j \leq j^{\prime} \leq m$ based on the algorithm in [19]. As analyzed in the latter, EXTEND takes $O\left(m^{2}\right)$ time. There are $O(n)$ free bases in $S_{1}$; thus, all calls to EXTEND require a total of $O\left(\mathrm{~nm}^{2}\right)$ time. The procedure MERGE will need to fill $O\left(\mathrm{~m}^{2}\right)$ entries in the combined table, each requires $O(m)$ time to compute because we need to find the maximum over $O(m)$ sums, in the worst case. Since MERGE is only invoked on arcs and the number of arcs in $P_{1}$ could reach $O(n)$; in total, all calls to MERGE require $O\left(\mathrm{~nm}^{3}\right)$ time. ARC-MATCH computes the term in Lemma 3 over $O\left(m^{2}\right)\left(j, j^{\prime}\right)$ pairs for each arc in $P_{1}$. Based on a similar argument on the number of arcs in $P_{1}$, ARC-MATCH requires $O\left(\mathrm{~nm}^{2}\right)$ time. As for the space requirement, assuming the standard traceback for inferring the secondary structure of the sequence $S_{2}$, we must store all intermediary DP tables computed by $W L C S\left(S_{1}, P_{1}, S_{2}\right)$. The cardinality of the latter is bounded by $O(n)$ as the number of free bases and arcs are both bounded by $O(n)$. In conclusion, the time and space complexity of the whole algorithm is $O\left(\mathrm{~nm}^{3}\right)$ and $O\left(\mathrm{~nm}^{2}\right)$, respectively.

\subsection{The Sparsification Technique - Monotonically Increasing Property of $D P$}

The previous section shows that the bottleneck of the computation of the WLCS score is in the procedure MERGE. Here, we describe how to speed up the computation of MERGE by taking advantage of the properties of $D P_{\left(i, i^{\prime}\right)}$.

Observation 1 For any $i \leq i^{\prime}, D P_{\left(i, i^{\prime}\right)}$ satisfies the following properties. 
1. In every row $j$ of $D P_{\left(i, i^{\prime}\right)}$, the entries are monotonically increasing, i.e., $D P_{\left(i, i^{\prime}\right)}\left[j, j^{\prime}\right] \leq D P_{\left(i, i^{\prime}\right)}\left[j, j^{\prime}+1\right]$.

2. In every column $j^{\prime}$ of $D P_{\left(i, i^{\prime}\right)}$, the entries are monotonically decreasing, i.e., $D P_{\left(i, i^{\prime}\right)}\left[j, j^{\prime}\right] \geq D P_{\left(i, i^{\prime}\right)}\left[j+1, j^{\prime}\right]$.

The observations above motivate the following definitions.

Definition 4. [5] For every row $j$ of $D P_{\left(i, i^{\prime}\right)}$, a position $j^{*}$ satisfying $j \leq j^{*} \leq$ $m$ is defined to be a row interval point if $D P_{\left(i, i^{\prime}\right)}\left[j, j^{*}-1\right]<D P_{\left(i, i^{\prime}\right)}\left[j, j^{*}\right]$. The set of row interval points $j^{*}$ in the $j^{\text {th }}$ row of $D P_{\left(i, i^{\prime}\right)}$ is denoted by $\operatorname{RowIP}_{j}\left(D P_{\left(i, i^{\prime}\right)}\right)$.

Definition 5. [5] For every column $j$ of $D P_{\left(i, i^{\prime}\right)}$, a position $j^{*}$ satisfying $1 \leq$ $j^{*} \leq j$ is defined to be a column interval point if $D P_{\left(i, i^{\prime}\right)}\left[j^{*}, j\right]>D P_{\left(i, i^{\prime}\right)}\left[j^{*}+\right.$ $1, j]$. The set of column interval points $j^{*}$ in the $j^{\text {th }}$ column of $D P_{\left(i, i^{\prime}\right)}$ is denoted by $\operatorname{ColIP}_{j}\left(D P_{\left(i, i^{\prime}\right)}\right)$.

Lemma 4. Let $\alpha=\max \left\{\beta, \alpha_{1}, \alpha_{2}\right\}$. Then there are at most $\left(\min \left\{\alpha\left(i^{\prime}-i+\right.\right.\right.$ $1),(m-j+1)\})$ row interval points in any row $j$ of $D P_{\left(i, i^{\prime}\right)}$.

Proof. (Sketch) The total number of row interval points (which are all distinct) in any row $j$ of $D P_{\left(i, i^{\prime}\right)}$ is bounded by the minimum of the maximum (integer) score and the number of columns in the row.

Corollary 1. There are at most $\left(\min \left\{\alpha\left(i^{\prime}-i+1\right), j^{\prime}\right\}\right)$ column interval points in any column $j^{\prime}$ of $D P_{\left(i, i^{\prime}\right)}$.

In [19], for every $\left(j, j^{\prime}\right)$ pair where $j \leq j^{\prime}$, the procedure $\operatorname{MERGE}\left(D P_{\left(i, i^{\prime}\right)}\right.$, $\left.D P_{\left(i^{\prime}+1, i^{\prime \prime}\right)}\right)$ tries every possible $j^{\prime \prime} \in\left[j-1 . . j^{\prime}\right]$ to compute the one that maximizes

$$
D P_{\left(i, i^{\prime}\right)}\left[j, j^{\prime \prime}\right]+D P_{\left(i^{\prime}+1, i^{\prime \prime}\right)}\left[j^{\prime \prime}+1, j^{\prime}\right]
$$

The following lemma states that it is unnecessary to consider all $j^{\prime \prime} \in\left[j-1 . . j^{\prime}\right]$ to find the maximum of $(1)$.

Lemma 5. The equation from Lemma 2 can be computed by

$$
\begin{aligned}
& D P_{\left(i, u_{r}\right)}\left[j, j^{\prime}\right]=
\end{aligned}
$$

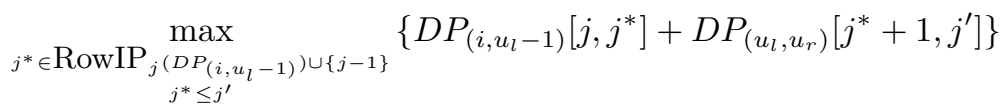

which checks at most $\left(\min \left\{\alpha\left(u_{l}-i\right)+1,\left(j^{\prime}-j+1\right)\right\}\right)$ candidates of $j^{*}$.

Proof. (Sketch) Let $F\left(j^{\prime \prime}\right)=D P_{\left(i, i^{\prime}\right)}\left[j, j^{\prime \prime}\right]+D P_{\left(i^{\prime}+1, i^{\prime \prime}\right)}\left[j^{\prime \prime}+1, j^{\prime}\right]$. By Lemma 2, $D P_{\left(i, u_{r}\right)}\left[j, j^{\prime}\right]=\max \left\{F(j-1), \max _{j^{\prime \prime} \in\left[j . . j^{\prime}\right]} F\left[j^{\prime \prime}\right]\right\}$. For each $j^{\prime \prime} \in\left[j . . j^{\prime}\right]$, we observe that there exists a $j^{*} \in \operatorname{RowIP}_{j}\left(D P_{\left(i, u_{l}-1\right)}\right)$ such that $j^{*} \leq j^{\prime \prime}$ and $D P_{\left(i, u_{l}-1\right)}\left[j, j^{*}\right]=D P_{\left(i, u_{l}-1\right)}\left[j, j^{\prime \prime}\right]$. Furthermore, since $j^{*} \leq j^{\prime \prime}$, we have $D P_{\left(u_{l}, u_{r}\right)}\left[j^{*}+1, j^{\prime}\right] \geq D P_{\left(u_{l}, u_{r}\right)}\left[j^{\prime \prime}+1, j^{\prime}\right]$. Hence, for such $j^{*}$ we have $F\left[j^{*}\right] \geq$ $F\left[j^{\prime \prime}\right]$ resulting in $\max _{j^{\prime \prime} \in\left[j . . j^{\prime}\right]} F\left[j^{\prime \prime}\right]=\max _{j^{*} \in \operatorname{RowIP}_{j}\left(D P_{\left(i, u_{l}-1\right)}\right)} F\left[j^{*}\right]$. 
Corollary 2. The equation from Lemma 2 can be computed by

$$
\begin{aligned}
& D P_{\left(i, u_{r}\right)}\left[j, j^{\prime}\right]= \\
& \max _{j^{*}+1 \in \operatorname{ColIP}_{\substack{j^{\prime}\left(D P\left(u_{l}, u_{r}\right) \\
j^{*}+1 \geq j\right.}}\left\{D j^{\prime}+1\right\}}\left\{D P_{\left(i, u_{l}-1\right)}\left[j, j^{*}\right]+D P_{\left(u_{l}, u_{r}\right)}\left[j^{*}+1, j^{\prime}\right]\right\}
\end{aligned}
$$

which checks at most $\left(\min \left\{\alpha|u|+1,\left(j^{\prime}-j+1\right)\right\}\right)$ candidates of $j^{*}$.

By Lemma 5 and Corollary 2, the time complexity of $\operatorname{MERGE}\left(D P_{\left(i, i^{\prime}-1\right)}\right.$, $\left.D P_{\left(i^{\prime}, i^{\prime \prime}\right)}\right)$ is improved to $O\left(\min \left\{\alpha\left(i^{\prime \prime}-i^{\prime}\right) m^{2}, \alpha\left(i^{\prime}-i\right) m^{2}, m^{3}\right\}\right)$.

\subsection{The Recursive Dynamic Programming Algorithm}

We now introduce a new algorithm $W L C S_{r}\left(S_{1}, P_{1}, S_{2}\right)$ which computes the table $D P_{(1, n)}$ using a carefully designed recursive dynamic programming algorithm. This improved algorithm guarantees that each MERGE operation is applied only on arcs whose size is at most half of its parent's ${ }^{1}$.

Let us start with some definitions. The followings are with respect to a nested annotated structure. An arc $u$ is a parent of an arc $v$ (denoted by Parent $(v)$ ) if $u_{l}<v_{l}<v_{r}<u_{r}$ and there is no arc $w$ such that $u_{l}<w_{l}<v_{l}<v_{r}<w_{r}<u_{r}$. Conversely, $v$ is referred as the child of the arc $u$. The set of children of an arc $u$ is denoted by Child $(u)$. A core-arc, with respect to an arc $u$, is a child of $u$ which has the biggest size (denoted as core-arc $(u)$ ). All other children of $u$ are named side-arcs and form the set side-arcs $(u)$. A terminal-arc is defined to be an arc which has no child. For any arc $u \in P_{1}$, the core-path $C P(u)$ is an ordered set of core-arcs $\left\{c_{1}, c_{2}, \cdots, c_{\ell}\right\}$, where $c_{1}=u$ and for any $c_{i}, c_{i+1}$ is core-arc $\left(c_{i}\right)$

$W L C S_{r}\left(S_{1}, S_{2}\right)$ first finds the largest arc $u$ in $[1 . . n]$ and processes every core$\operatorname{arc} c \in C P(u)$ from the innermost to the outermost. For terminal $\operatorname{arcs} t, D P_{\left(t_{l}, t_{r}\right)}$ can be computed by using EXTEND operations only. For the remaining $\operatorname{arcs} c$, $D P_{\left(c_{l}, c_{r}\right)}$ is obtained using a two-part computation. Let $c^{\prime}$ be core-arc $(c)$. Due to the bottom-up ordering, $D P_{\left(c_{l}^{\prime}, c_{r}^{\prime}\right)}$ will have been computed at this point of time. We first compute the value of $D P_{\left(c_{l}+1, c_{1}^{\prime}-1\right)}$ (the LEFT Part phase) using EXTEND and MERGE operations. Given $D P_{\left(c_{l}+1, c_{l}^{\prime}-1\right)}$, we proceed using EXTEND and MERGE to compute $D P_{\left(c_{l}^{\prime}, c_{r}-1\right)}($ the RIGHT Part phase). In both phases, whenever we encounter a side-arc $s$, we first compute $D P_{\left(s_{l}, s_{r}\right)}$ by recursively calling $W L C S_{r}\left(S_{1}\left[s_{l} . . s_{r}\right], S_{2}\right)$. Next, we apply MERGE on $D P_{\left(c_{l}+1, c_{l}^{\prime}-1\right)}$ and $D P_{\left(c_{l}^{\prime}, c_{r}-1\right)}$ to compute $D P_{\left(c_{l}+1, c_{r}-1\right)}$. Finally, $D P_{\left(c_{l}, c_{r}\right)}$ is obtained by applying ARC-MATCH $\left(D P_{\left(c_{l}+1, c_{r}-1\right)}\right)$. If $(1, n) \in P_{1}$, then $u=(1, n)$ and we are done. Otherwise, we need to compute $D P_{(1, n)}$ using the same two-part

\footnotetext{
${ }^{1}$ The routine $W \operatorname{LCS}\left(S_{1}, P_{1}, P_{2}\right)$ given in [19] computes the DP tables according to the postorder of the nodes in their tree representation. The problem of this approach is that we may need to perform MERGE on arcs with large sizes causing an $\Omega\left(n m^{2}\right)$ space requirement even if we only wish to compute the WLCS score of $\left(S_{1}, P_{1}\right)$ and $S_{2}$. We shall prove this claim in the full version of this paper.
} 


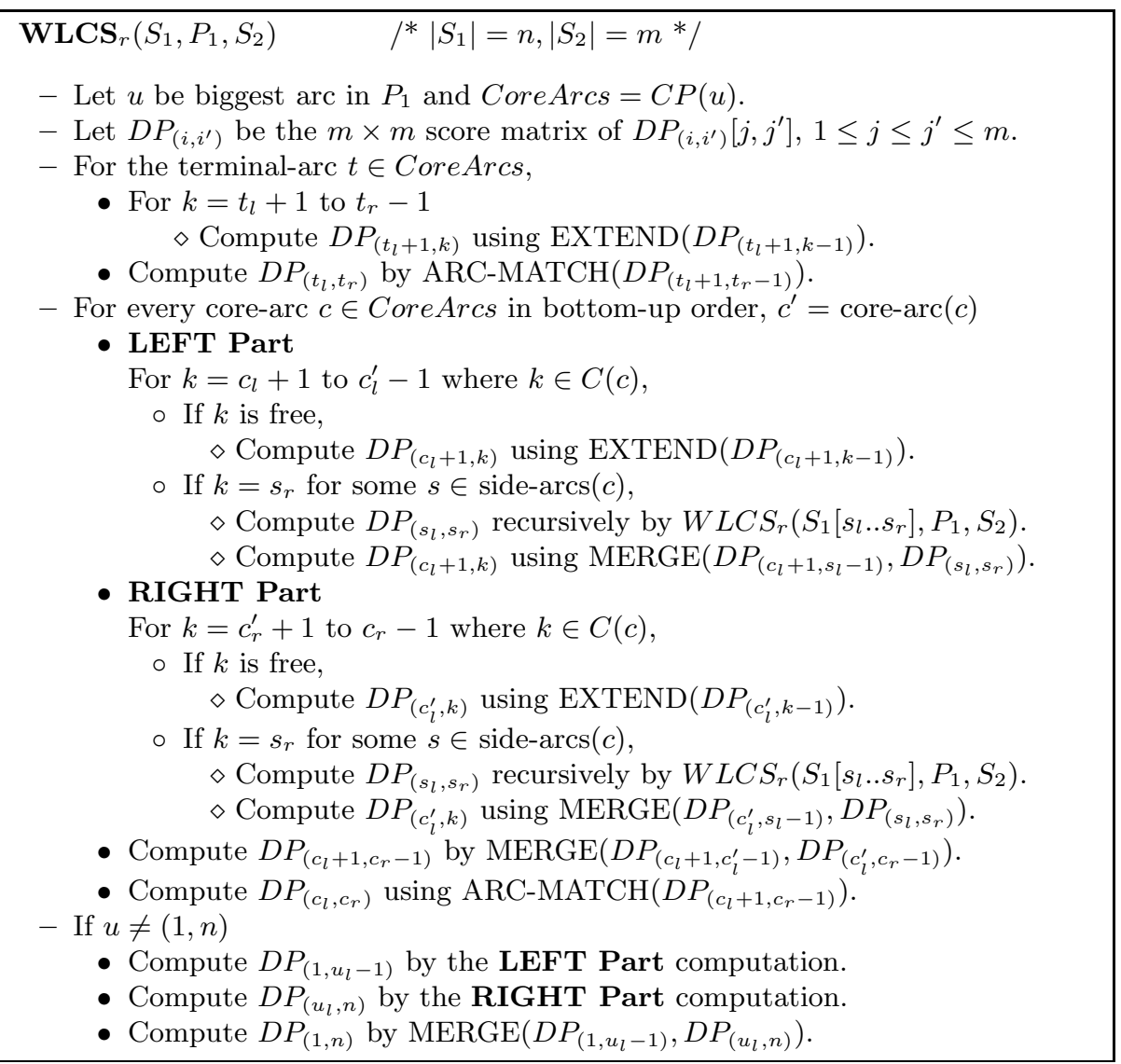

Fig. 2. The algorithm $W L C S_{r}\left(S_{1}, P_{1}, S_{2}\right)$.

computation technique: first compute $D P_{\left(1, u_{l}-1\right)}$, followed by $D P_{\left(u_{l}, n\right)}$, and then obtain $D P_{(1, n)}$ by $\operatorname{MERGE}\left(D P_{\left(1, u_{l}-1\right)}, D P_{\left(u_{l}, n\right)}\right)$. Our complete algorithm $W L C S_{r}\left(S_{1}, P_{1}, S_{2}\right)$ is listed in Fig. 2.

Lemma 6. $W L C S_{r}\left(S_{1}, P_{1}, S_{2}\right)$ runs in $\min \left\{O\left(\alpha n m^{2} \log n\right), O\left(n m^{3}\right)\right\}$ time.

Proof. To obtain the execution time of $\operatorname{WLC} S_{r}\left(S_{1}, P_{1}, S_{2}\right)$, we analyze the total execution time of the EXTEND, MERGE, and ARC-MATCH operations separately. The time required by EXTEND and ARC-MATCH operations is still the same as in [19], namely $O\left(\mathrm{~nm}^{2}\right)$, as they are still applied at most once on every free bases and arcs, respectively. Note that MERGE is now invoked on all arcs which belong to the set $\operatorname{side}-\operatorname{arc}(u)$ for some $\operatorname{arc} u \in P_{1}$ and on the merging of the LEFT part and the RIGHT part of all non-terminal arcs. For any side-arc $s$, merging the table $D P_{\left(s_{l}, s_{r}\right)}$ into some table $D P_{\left(i, s_{l}-1\right)}$ takes at most $O\left(\min \left\{\alpha|s| m^{2}, \alpha\left(s_{l}-i\right) m^{2}, m^{3}\right\}\right)$ which is at most $O\left(\min \left\{\alpha|s| m^{2}, m^{3}\right\}\right)$ time. In the second type of MERGE invocations, we execute MERGE $\left(D P_{\left(c_{l}+1, c_{l}^{\prime}-1\right)}\right.$, 
$\left.D P_{\left(c_{1}^{\prime}, c_{r}-1\right)}\right)$ for all non-terminal $\operatorname{arcs} c$ where $c^{\prime}=$ core-arc $(c)$. The latter requires $O\left(\min \left\{\alpha\left(c_{l}^{\prime}-c_{l}\right) m^{2}, \alpha\left(c_{r}-c_{l}^{\prime}\right) m^{2}, m^{3}\right\}\right) \leq O\left(\min \left\{\alpha\left(c_{l}^{\prime}-c_{l}\right) m^{2}, m^{3}\right\}\right)$. Let $r$ be an imaginary arc where $r=(0, n+1)$ and $T\left(S_{1}\right)$ be the total execution time for all MERGE operations in $W L C S_{r}\left(S_{1}, P_{1}, S_{2}\right)$.

$$
\begin{aligned}
T\left(S_{1}\right)= & \sum_{c \in C P(r)}\left(\sum_{s \in \operatorname{side-arcs}(c)} T\left(S_{1}\left[s_{l} . . s_{r}\right]\right)+O\left(\min \left\{\alpha|s| m^{2}, m^{3}\right\}\right)+\right. \\
= & \sum_{c \in C P(r)} O\left(\min \left\{\alpha\left(c_{l}^{\prime}-c_{l}\right) m^{2}, m^{3}\right\}\right) \\
& \sum_{\substack{s \in \operatorname{side-arcs}(c) \\
c \in C P(r)}} T\left(S_{1}\left[s_{l} . . s_{r}\right]\right)+\sum_{\substack{s \in \operatorname{side-arcs}(c) \\
c \in C(r)}} O\left(\min \left\{\alpha|s| m^{2}, m^{3}\right\}\right)+ \\
= & \sum_{\substack{c \in C P(r) \\
s \in \operatorname{side-arcs}(c)}} T\left(\min \left\{\alpha\left(c_{l}^{\prime}-c_{l}\right) m^{2}, m^{3}\right\}\right)
\end{aligned}
$$

Both the second and the third summation terms in (3) sum up to $O\left(\min \left\{\alpha n m^{2}\right.\right.$,

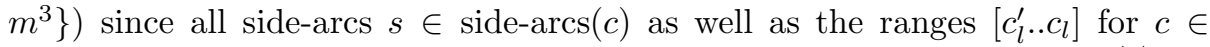
$C P(r)$ are non overlapping. Based on the fact that $\sum|s| \leq|c|$ and $|s| \leq \frac{|c|}{2}$, by inspection, the solution of the recurrence is $O\left(\alpha n m^{2} \log n\right)$ if $\min \left\{\alpha n m^{2}, m^{3}\right\}=$ $\alpha \mathrm{nm}^{2}$ or $O\left(\mathrm{~nm}^{3}\right)$ otherwise. Combining the running time of the three operations, the lemma follows.

\section{Traceback Using a Hirschberg-Based Technique}

Using the standard traceback algorithm, one is required to store all $D P$ tables corresponding to any arc $u \in P_{1}$. Alternatively, we can make use of the recursive technique introduced by Hirschberg in [10] and use $W L C S_{r}\left(S_{1}, P_{1}, S_{2}\right)$ only to compute the WLCS score. We shall refer to the latter as the score-only $W L C S_{r}\left(S_{1}, P_{1}, S_{2}\right)$.

Lemma 7. Computing the score-only $W L C S_{r}\left(S_{1}, P_{1}, S_{2}\right)$ requires $\min \left\{O\left(m^{2} \log n\right), O\left(m^{2}+\alpha m n\right)\right\}$ space.

Proof. To compute the score-only $W L C S_{r}\left(S_{1}, P_{1}, S_{2}\right)$, since we do not have to traceback, we can just store the information needed to compute the alignment score. This corresponds to $O\left(\mathrm{~m}^{2}\right)$ space for the EXTEND and ARCMATCH operations. As for the MERGE operations, when there is no recursive call involved (the second type of MERGE), the space requirement is also in $O\left(\mathrm{~m}^{2}\right)$. Otherwise, referring back to Fig. 2, when we invoke the recursive call $W L C S_{r}\left(S_{1}\left[s_{l} . . s_{r}\right], P_{1}, S_{2}\right)$, we observe that we need to store $D P_{\left(i, s_{l}-1\right)}$ for some fixed $i$. Storing only the row interval points takes $O\left(\min \left\{\alpha\left(s_{l}-i\right) m, m^{2}\right\}\right)$ space (by Lemma 4). Since recursive calls are only applied on side-arcs, we have at 


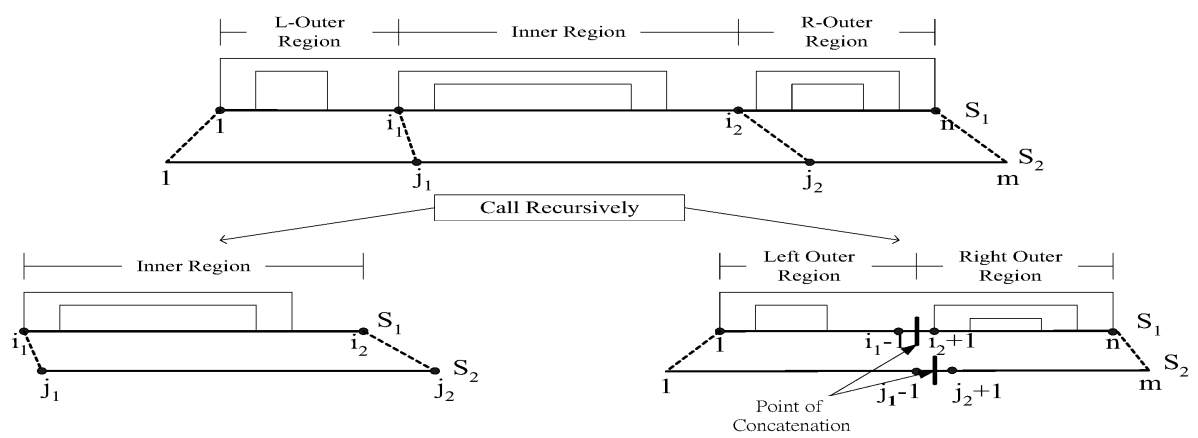

Fig. 3. The recursion on the partitioned continuous region by Lemma 8 .

most $O(\log n)$ recursion levels giving an upper bound of $O\left(m^{2} \log n\right)$ on the space complexity. We further claim that the space required is smaller than $O(\alpha n m)$ since, in each recursion level $x$, we only store $D P_{\left(i_{x}, s_{l_{x}}-1\right)}$ where all of the intervals $\left[i_{x} . . s_{l_{x}}-1\right]$ are disjoint. Hence, $\sum_{x} O\left(\alpha\left(s_{l_{x}}-i_{x}\right) m\right) \leq O(\alpha n m)$. Combining the three terms, the lemma follows.

Following the idea of Hirschberg in [10], we compute the WLCS alignment between $\left(S_{1}, P_{1}\right)$ and $S_{2}$ as follows,

1. Divide $S_{1}$ into a constant number of non-overlapping regions $S_{11}, S_{12}, . . S_{1 c}$.

2. For each region $S_{1 i}$, find the region $S_{2 i}$ in $S_{2}$ such that the optimal WLCS alignment will align $S_{1 i}$ to $S_{2 i}$.

3. Recursively compute the optimal WLCS alignments between $S_{1 i}$ and $S_{2 i}$ for $i=1,2, . ., c$.

To do the first step, since $S_{1}$ is arc-annotated, we must divide $S_{1}$ in such a way that we do not break any arc in $P_{1}$. The solution is to divide $S_{1}$ into inner and outer regions. Given two points $i_{1}$ and $i_{2}, 1 \leq i_{1} \leq i_{2} \leq n$, the inner region with respect to $i_{1}$ and $i_{2}$ is $S_{1}\left[i_{1} . . i_{2}\right]$ and the outer region is the concatenation of $S_{1}\left[1 . .\left(i_{1}-1\right)\right]$ and $S_{1}\left[\left(i_{2}+1\right) . . n\right]$ (see Fig. 3$)$. The latter is also referred as a gapped region since it has a discontinuous interval $\left(S_{1}\left[i_{1} . . i_{2}\right]\right.$ is removed). Let $\star$ be a special character that denotes the gap in the sequence such that the gapped region can be written as $S_{1}\left[1 . .\left(i_{1}-1\right)\right] \star S_{1}\left[\left(i_{2}+1\right) . . n\right]$. If a region has no gap in it, we say it is continuous. We shall show that we can bound the size of each region by $\phi n$ for some constant $\phi, 0<\phi<1$. Due to space constraints, the proofs of the following lemmas will appear in the journal version of this paper.

Lemma 8. We can always partition a continuous region into 2 non-overlapping subregions, where one of them is continuous and the other is gapped. Every subregion's size is at most $\frac{2}{3}$ of the original region.

Lemma 9. We can always partition a gapped region into at most 4 non-overlapping subregions, where at most one of them is continuous. Every subregion's size is at most $\frac{2}{3}$ of the original region. 
After dividing $S_{1}$ into at most 4 subregions, where each is denoted by $S_{1 i}$ for $i \leq 4$, we now need to compute the regions $S_{2 i}$ in $S_{2}$ to which the subregions $S_{1 i}$ is aligned by the optimal WLCS alignment.

Lemma 10. For any $1 \leq i_{1} \leq i_{2} \leq n$, we can compute $1 \leq j_{1} \leq j_{2} \leq m$, such that the optimal alignment between $\left(S_{1}, P_{1}\right)$ and $S_{2}$ aligns $S_{1}\left[i_{1} . . i_{2}\right]$ to $S_{2}\left[j_{1} . . j_{2}\right]$, within the same time and space complexity of the score-only $W L C S_{r}\left(S_{1}, P_{1}, S_{2}\right)$.

Lemma 11. For any $1 \leq i_{1} \leq i_{2} \leq i_{3} \leq i_{4} \leq n$, we can compute $1 \leq j_{1} \leq j_{2} \leq$ $j_{3} \leq j_{4} \leq m$, such that the optimal alignment between $\left(S_{1}, P_{1}\right)$ and $S_{2}$ aligns $S_{1}\left[i_{1} . . i_{2}\right] \star S_{1}\left[i_{3} . . i_{4}\right]$ to $S_{2}\left[j_{1} . . j_{2}\right] \star S_{2}\left[j_{3} . . j_{4}\right]$, within the same time and space complexity of the score-only $W L C S_{r}\left(S_{1}, P_{1}, S_{2}\right)$.

By Lemmas 10 and 11, the second step of this algorithm can be executed within the same time and space complexity of the score-only $W L C S_{r}\left(S_{1}, P_{1}, S_{2}\right)$ since the number of the subregions is constant. Next, we proceed by applying the algorithm recursively on each pair $\left(S_{1 i}, S_{2 i}\right)$. While applying the algorithm on the continuous region is straightforward, the gapped region needs a bit of extra care. In this case, $\star$ in $S_{1 i}$ must be aligned to $\star$ in $S_{2 i}$ because they represent the subregion pair(s) computed in the other recursive call(s). To implement such constraint, we add into the base scoring function the following cases: $\chi(\star, \star)=0$ and $\chi(\star, x)=\chi(x, \star)=-\infty$ for $x \in\{A, C, G, U, \sqcup\}$. This way, the optimal alignment between the two sequences is forced to align $\star$ in the first sequence to $\star$ in the second in order to have a non-negative score.

Lemma 12. Our new algorithm can recover the optimal WLCS alignment in $\min \left\{O\left(\alpha n m^{2} \log n\right), O\left(n m^{3}\right)\right\}$ time and $\min \left\{O\left(m^{2} \log n\right), O\left(m^{2}+\alpha m n\right)\right\}$ space.

\section{Concluding Remarks}

Consider two homologous RNA sequences $S_{1}$ and $S_{2}$ where $S_{1}$ has a known structure. This paper presents an improved algorithm to solve the problem of inferring the structure of $S_{2}$ such that the WLCS score between the two structures are maximized. The same algorithm can easily be applied to the longest arc-preserving common subsequence problem (LAPCS) (see, e.g., $[4,11])$. In particular, we improve the time and space complexity of LAPCS (nested, plain) problem from $O\left(n m^{3}\right)$ and $O\left(n m^{2}\right)[11]$ to $\min \left\{O\left(n m^{2} \log n\right), O\left(n m^{3}\right)\right\}$ and $\min \left\{O\left(m^{2}+m n\right), O\left(m^{2} \log n\right)\right\}$.

One interesting extension of the problem discussed in this paper is to incorporate a more realistic, non-linear scoring function into the base and arc matching function. Another possible direction is to attempt some special cases of crossed arc-annotation structures, which can represent pseudoknotted structures in RNA sequences, by applying the algorithm iteratively.

\section{References}

1. J. Alber, J. Gramm, J. Guo, and R. Niedermeier. Towards optimally solving the longest common subsequence problem for sequences with nested arc annotations in linear time. In $C P M$, pages 99-114, 2002. 
2. V. Bafna, S. Muthukrishnan, and R. Ravi. Computing similarity between RNA strings. In $C P M$, volume 937, pages 1-16, 1995.

3. R.B. Carey and G.D. Stormo. Graph-theoretic approach to RNA modeling using comparative data. In $I S M B$, pages 75-80, 1995.

4. P. A. Evans. Algorithms and Complexity for Annotated Sequence Analysis. PhD Thesis, University of Victoria, 1999.

5. W. Fu, W. K. Hon, and W. K. Sung. On all-substrings alignment problems. In COCOON, volume 2697, pages 80-89, 2003.

6. J. Gramm, J. Guo, and R. Niedermeier. Pattern matching for arc-annotated sequences. In FSTTCS, volume 2556, pages 182-193, 2002.

7. L. Grate, M. Herbster, R. Hughey, I. S. Mian, H. Noller, and D. Haussler. RNA modeling using Gibbs sampling and stochastic context free grammars. In ISMB, pages 138-146, 1994.

8. L. Grate. Automatic RNA secondary structure determination with stochastic context-free grammars. In ISMB, pages 136-144, 1995.

9. R.R. Gutell, N. Larsen, and C.R. Woese. Lessons from an evolving rRNA: $16 \mathrm{~S}$ and 23S rRNA structures from a comparative perspective. Microbiological Reviews, 58(1):10-26, 1994.

10. D. S. Hirschberg. Algorithms for the longest common subsequence problem. J. Association of Computing Machinery, 24(4):664-675, 1977.

11. T. Jiang, G. H. Lin, B. Ma, and K. Zhang. The longest common subsequence problem for arc-annotated sequences. In CPM, volume 1848, pages 154-165, 2000. To appear in Journal of Discrete Algorithms.

12. D.A.M. Konings and R.R. Gutell. A comparison of thermodynamic foldings with comparatively derived structures of 16s and 16s-like rRNAs. RNA, 1:559-574, 1995.

13. G. H. Lin, Z. Z. Chen, T. Jiang, and J. Wen. The longest common subsequence problem for sequences with nested arc annotation. Journal of Computer and System Sciences, 65:465-480, 2002.

14. G. H. Lin, B. Ma, and K. Zhang. Edit distance between two RNA structures. In RECOMB, pages 211-200, 2001.

15. R. B. Lyngs $\varnothing$, M. Zuker, and C.N.S. Pedersen. Internal loops in RNA secondary structure prediction. In RECOMB, pages 260-267, 1999.

16. R. Nussinov and A.B. Jacobson. Fast algorithm for predicting the secondary structure of single stranded RNA. In PNAS, volume 77(11), pages 6309-6313, 1980.

17. Y. Sakakibara, M. Brown, R. Hughey, I.S. Mian, K. Sjölander, R.C. Underwood, and D. Haussler. Recent methods for RNA modeling using stochastic contextfree grammars. In Proc. of the Asilomar Conference on Combinatorial Pattern Matching, 1994.

18. J.E. Tabaska, H.N. Gabow R.B. Cary, and G.D. Stormo. An RNA folding method capable of identifying pseudoknots and base triples. Bioinformatics, 14(8):691-699, 1998.

19. K. Zhang. Computing similarity between RNA secondary structures. In IEEE International Joint Symposia on Intelligence and Systems, pages 126-132, 1998.

20. M. Zuker. Prediction of RNA secondary structure by energy minimization. In Methods in Molecular Biology, volume 25, pages 267-94, 1994.

21. M. Zuker and P. Stiegler. Optimal computer folding of large RNA sequences using thermodynamics and auxiliary information. Nucleic Acid Res. 9, pages 133-148, 1981. 